\title{
Computer Analysis of Smoke Protection of an Atrium Building in a French Business Centre
}

\author{
XAVIER EMILE BODART, MICHEL ROGER CURTAT and \\ PHILIPPE GEORGES FROMY \\ Centre Scientifique et Technique du Bâtiment \\ B.P. No. 02 \\ 77421 Marne La Vallée Cedex 2, France
}

\begin{abstract}
In France, the design of smoke control systems for buildings open to the public commonly depends on regulatory requirements on minimal values of smoke vent areas for each building space, in natural ventilation.

These regulations proved unsatisfactory for atrium buildings, and in some cases building designers as well as safety authorities must be provided with specific means for designing a smoke control system and evaluating its efficiency.
\end{abstract}

This paper gives an illustration of what computer analysis can bring for this purpose, through the example of an atrium building in a new business centre near Paris (CNIT / La Défense).

Simulations of smoke movement throughout this 5-storey building containing an 18-metre high atrium were carried out with the csmB multiroom computer code CIEI.

The roughness of zonal description in the model is balanced by the capability of treating the physical system as a whole for the computer simulations. Consequently, the analysis allowed us to draw relevant information from simulation results, and then contributed to the elaboration of a reasonable agreement resulting in the acceptance of the project (the business centre has been recently inaugurated).

KEYWORDS: Fires - Atrium - Smoke movement - Smoke control Zone model - 


\section{REGULATORY REQUIREMENTS AND ATRIUM BUILDINGS}

French regulations on smoke control with natural ventilation in buildings "open to the public" are generally unsuitable to the case of atria, since they use formulae and tables (1) that lead to unrealistic values of minimal smoke vent areas for high atria and/or small atrium floor areas. Some authors have pointed out weaknesses in underlying assumptions of regulatory calculations (2), whilst some do not believe in the possibility of standardization for fire safety design in atria (3), unlike others (4).

More modestly, we are devoting the following to the presentation of a specific case of atrium building in France, for which a computer analysis performed at CSTB contributed to establish an agreement between the building designer and the safety authorities, concerning the definition of a smoke protection system which could not have been satisfactorily reached through a mere application of our standard regulations.

\section{THE PROBLEM OF SMOKE CONTROL IN THE CNIT ATRIUM BUILDING}

The CNIT building was erected during the fifties in La Defense, a western suburban district of Raris sometimes called "little Manhattan". Basically it consisted of a triangle-shaped reinforced concrete shell, symmetric, whose vertices are as if bent downwards in such a way that the shell, which is the roof of the building, appears to rest at ground level on its three vertices, and its three sides are arc-shaped. These sides delimit the upper edges of three equally huge window bays (more than $200 \mathrm{~m}$ longl that were totally glazed. The CNIT building has been used under this form for more than 30 years, during which its pure lignes and its immense internal volume greeted numerous and various exhibitions and events.

Recently a redevelopment process took place in order to transform the CNIT into an international business centre fully equipped with peripherical facilities (hotels, restaurants, shopping and relaxing units ...). For this purpose, whereas the concrete shell itself was kept, parts of the glazing were removed or modified in order to let new construction erected under the shell extend beyond initial façades.

The CNIT atrium building is thus one of these new buildings which are located mainly under the initial concrete shell and partly outside the main construction (see plane view of Figure 1): its name is INFOMART, as it contains a number of show-rooms covering activities of informatics trade. This is a 5 -level building (see vertical section of Figure 2), and in the middle there is a large atrium volume: $100 \mathrm{~m}$ long, $10 \mathrm{~m}$ wide and $\bar{\sim} 18 \mathrm{~m} \mathrm{high}$.

When CSTB got involved in the safety design problem, the designer proposed a hybrid system for the ventilation in case of fire:

(i) fan-powered exhaust system capable of drawing smoke from upper parts of all show-rooms: $1 \mathrm{~m}^{3}$ per second per $100 \mathrm{~m}^{2}$ floor area (which is close to an average value of show-room floor areas)

(ii) fan-powered supply system for bringing fresh air to lower parts of all walkways at each level (ground level included): on Figure 2, walkways separating show-room spaces from atrium space are visible. The global rate of outside air that can be blown through the system is about $80000 \mathrm{~m}^{3}$ per hour, equally dispatched amongst all levels 
(iii) natural air supply to the bottom of the atrium through doors (area $30 \mathrm{~m}^{2}$ )

(iv) smoke vents at the top of the atrium: area $\bar{\sim} 50 \mathrm{~m}^{2}$.

The value given in (i) is in accordance with French regulations for this type of building. The value given in (iv) is consistent with temporary requirements (5) for smoke vents in atria, i.e. 1/20th of the atrium floor area. The value given in (ii) and (iii) ensure that the flow rate of air supply to the atrium volume is equivalent to what could flow through a cross section of $50 \mathrm{~m}^{2}$, with a standard average velocity of $1 \mathrm{~m} / \mathrm{s}$.

Besides, the showroom volume is fully sprinkled and the whole building is equipped with smoke detectors.

The main concern of authorities having to accept or refuse the project on fire safety considerations resulted from ignorance about ambient conditions likely to be encountered by occupants and/or firemen during an egress/intexvention process following a fire outbreak.

our feeling was that the above-mentioned data, referring only to standard and regulatory considerations, had given almost no information about real processes of interaction between fire development / smoke propagation and ventilation system operation. Therefore, we proposed to the designers and the authorities to perform computer simulations of what might happen in the atrium building after a fire outbreak. However, the ventilation system likely to exhaust smoke from show-rooms appeared evidently unable to

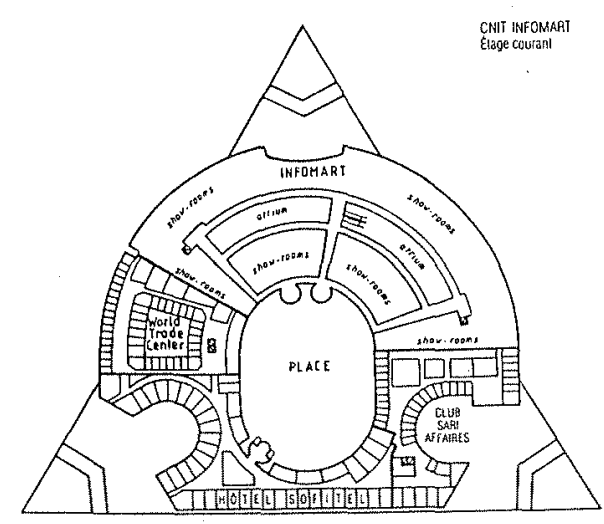

FIGURE 1

Plane view (horlzontal section) of the CNIT bullding, were INFOMART building is located. Note that each side of the trangle is more than $200 \mathrm{~m}$ long.

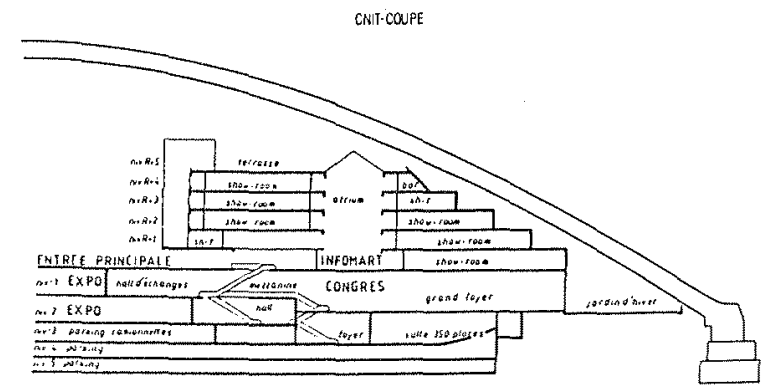

PIGURE 2

Side view (vertical section) of INFOMART building, within the CNIT 
counterbalance a substantial production of smoke, and the mechanical air supply to walkways appeared as well unable to set a pressure barrier capable of preventing smoke propagation from the atrium space to walkways through balconies. Thus, two phenomena seemed a priori possible in case of fire in a show-room:

- smoke propagation from this show-room to atrium space through the intermediate walkway,

- smoke propagation from atrium to walkways through balconies at some other levels of the building.

\section{COMPUTER STMULATIONS OF FIRE-INDUCED SMOKE MOVEMENT THROUGHOUT THE CNIT ATRIUM}

A first set of computer simulations aimed at assessing the likelihood of smoke propagation from a show-room to the walkway next to it.

A complete description of configuration data is given in (6). The computer code used for the simulation is FISBA, which has been developed at CSTB for several years within the framework of the French research programme EIS (Feu, Incendie, Securité) (7-10). The mathematical model starts from a description of the physical system assuming gas stratification within the room; heat and mass transfer is then written and balanced for each of the zones taken into account, $i . e$. the two gaseous layexs and wall zones: thus, EISBA is a finite volume model, not a field model. Plume equations used for the calculation of entrained air over the fire bed depend on scenario: zUKOSKI formulae (11) were chosen for small and medium fires, THOMAS' (12) for big fires.

The main data are:

- geometry of the show-room: floor area of $90 \mathrm{~m}^{2}$ and $3 \mathrm{~m}$ in height

- show-room /outside door, always open: width of $1.40 \mathrm{~m}$ and $2 \mathrm{~m}$ in height

- smoke exhaust outlet located at $2.70 \mathrm{~m}$ over the floor, exhaust flow rate is $0.9 \mathrm{~m}^{3} / \mathrm{s}$ for $100 \mathrm{~m}^{2}$ floor area)

- the fan-powered smoke exhaust system is always off, or is started 30 seconds after the beginning of the fire

- fire scenarios are as follows: combustible is wood and pyrolysis mass flow rate rises quasi-innearly from zero to a plateau. The value at this plateau is:

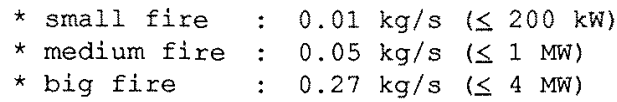

(NB: for small and medium fires, slightly higher values of heat of combustion per mass unit are used).

The plateau is reached after $5 \mathrm{~min}$ or after $15 \mathrm{~min}$ (this varies from case to case). After $t=20 \mathrm{~min}$ the pyrolysis flow rate drops.

On Figure 3 , the calculated volume flow rates of smoky gases leaving the show-room through the door are plotted for 4 scenarios involving a big fire: 
- in cases \# 1 and \# 2 the pyrolysis plateau is reached after 5 min

- in cases \#3 and \#4 it is reached after $15 \mathrm{~min}$

- in cases \#1 and \#3 the fan-powered exhaust system is always off

- in cases \#2 and \#4 it operates after 30 s as stated above.

It can be seen that the operation of the smoke exhaust fanpowered system has almost no influence on smoke propagation from show-room to other volumes lonly a reduction of less than $10 \frac{0}{5}$ of flow rates can be observed). As the data for the "big fire" correspond to what authorities consider as a "regulatory fire" for this class of building, this set of simulations establishes the relevance of a study foreseeing scenarii of smoke spread from initial fire zones in a show-room, throughout the whole atrium building.

However, other predictions with various fire sizes were calculated in this first set of simulations ( 6$)$ : they showed that the fanpowered smoke exhaust system was always efficient for small fires and "halfefficient" for medium fires.

On Figure 4 is drawn a typical sketch of the configurations dealt with for the computer simulations performed with the CSTB multiroom smoke movement computer code CIFI (8), which generalizes FISBA model to multiroom configurations, as two gaseous layers can be considered for each room. Doorjet entrainment raises problems that were extensively examined by BODART and CURTAT (9), and rates have been calculated here in accordance with their proposal of getting estimates by using zUKOSKI formulae valid for far field regions of fire plumes. As to air entrainment in fire plumes themselves, however, THoMAs formulae were used (12) for these simulations. Other important data also used are as follows:

- show-room where fire development and smoke production takes place: height of $3 \mathrm{~m}$ and floor area of 90 or $180 \mathrm{~m}^{2}$, location at ground level of the building in most scenarii

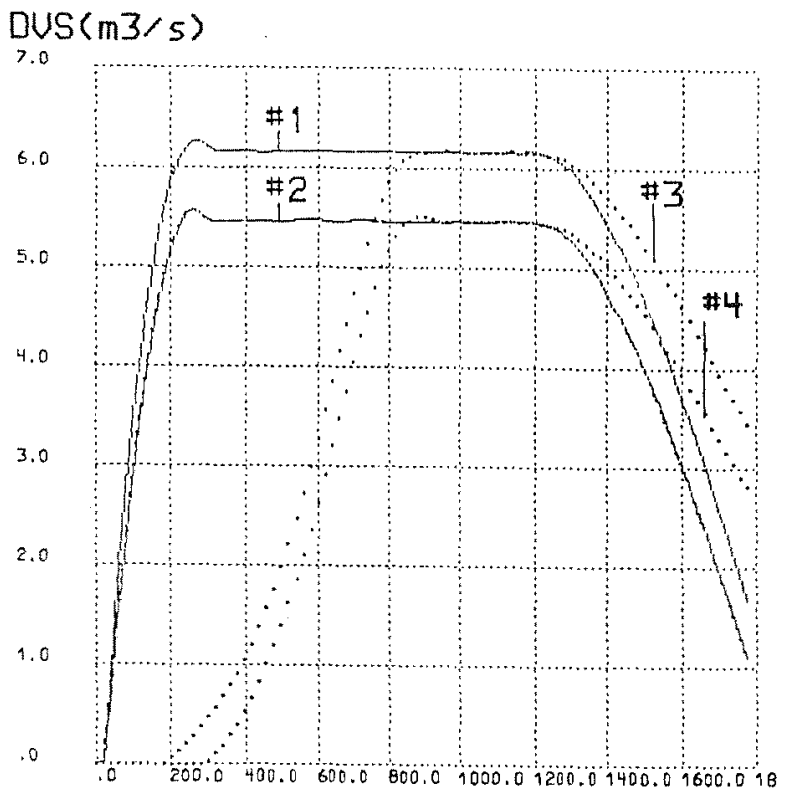

$t(s)$

Volume flow rates of smoky gases leaving the showroom, calculated with FISBA code for 4 scenaril of

fire/fan power operation. 
VENT $=0.0 \mathrm{~ms}$

cw $=1.00 \quad 0.00-1.00 \quad 0.00 \quad 0.00$

$T E X=30.0030 .0030 .0030 .0030 .00$

debit ref. $=49.789 \mathrm{~kg}^{\prime} \mathrm{s}$
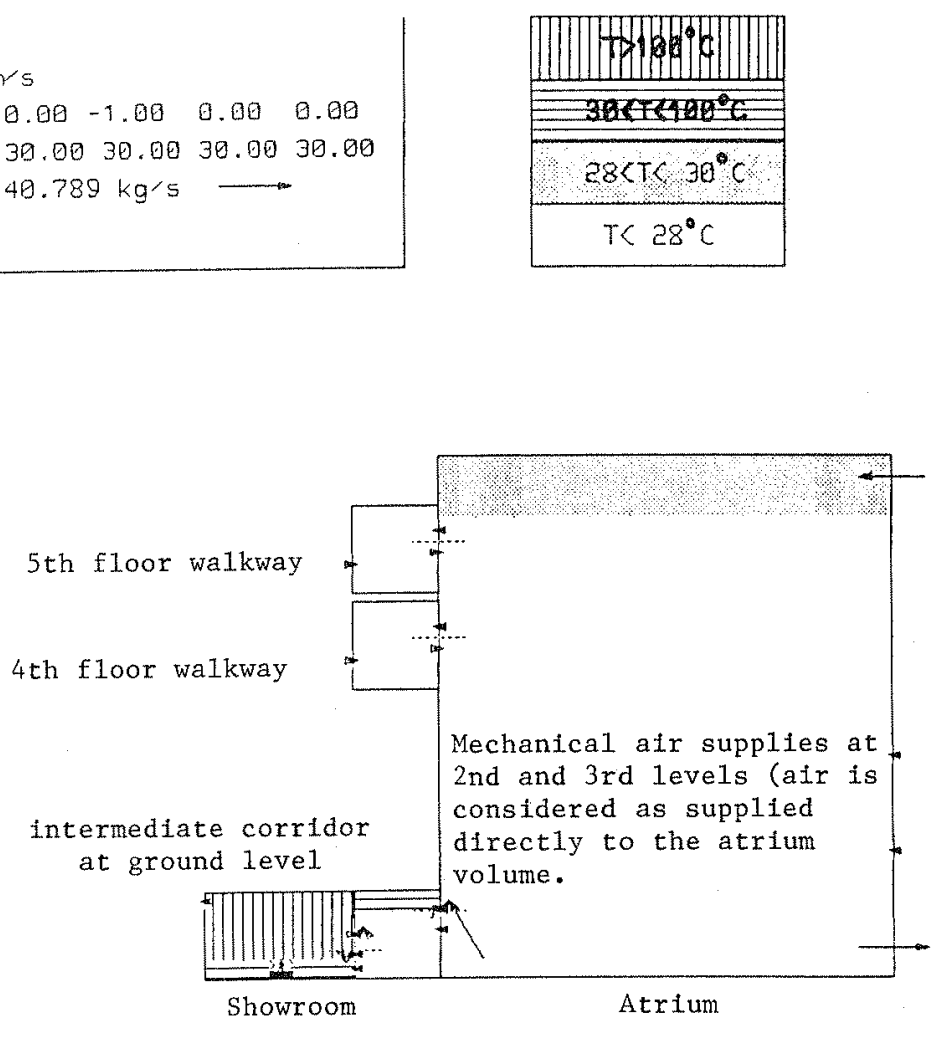

TEMPS $=90.00$ SECONDES

\section{FIGURE 4}

Sketch of amblent conditions calculated with CIFI code in the multiroom configuration, and examined $90 \mathrm{~s}$ after a fire outbreak. This is a vertical cross-section of the set of 5 volumes considered for all the simulations. Each volume consists of 1 or 2 gaseous zones as may be seen above. Vartous arrows refer to mass flow rates from one zone to another; some of them cannot be distinguished clearly, but the longest ones are highly visible: they refer to air entrained into the atrium plume and natural ventilation to the top and from the bottom of the atrium. The first two lines of top

left captions mean there is no wind, the third line that the outside temperature is $30^{\circ} \mathrm{C}$, the fourth line gives a reference scale for rate arrows. 
- part of a walkway next to that show-room (length of $45 \mathrm{~m}$ in a transverse direction and width of $2.4 \mathrm{~m}$ ); this part of walkway was chosen as going along 2 show-rooms on either side of the initialshowroom

- atrium volume: $100 \mathrm{~m}$ long, $10 \mathrm{~m}$ large, $17.9 \mathrm{~m} \mathrm{high}$

- walkways on the 4 th and 5 th levels: width of $2.4 \mathrm{~m}$ and length of $200 \mathrm{~m}$ (equivalent to $100 \mathrm{~m}$ on either side of atrium volume)

- outside doors of the atrium have been already mentioned, as well as smoke vents located in the upper part of this space. The former are always open, the latter are closed initially, opened at $30 \mathrm{~s}$ after the beginning of the fire

- balcony openings between atrium space and walkways: $1.5 \mathrm{~m}$ high and as large as the walkways themselves

- fan-powered supply and exhaust system have been already mentioned: either they are started at 30 s or they do not operate

- mechanical air supply is equally distributed amongst the 5 levels, except that of fire outbreak, i.e. amongst 4 levels. As a minimal set of volumes was taken into account (in order to avoid too long CPU times), fan-powered air supply is assumed to take place in the atrium space itself on levels where walkways are not described (i.e. generally on the 2nd and 3rd levels, as the level of fire outbreak is the first level in most cases)

- two sets of ambient conditions before the beginning of a fire were taken into account:

* winter conditions: 5 deg.C outside, 15 deg.C in the atrium space and along the walkways, $19 \mathrm{deg} . \mathrm{C}$ in the show-room

* summer conditions: respectively 30 deg.C, 26 deg.C, 25 deg.c

on Figure 4, with summer conditions, $90 \mathrm{~s}$ after a fire outbreak, the calculated ambient conditions are as follows:

- the show-room (bottom left) is almost totally logged with smoke (vertical hatching)

- the 1st level walkway is smoke-logged in its upper part, down to the bottom of the vertical screen which is also the upper limit of the balcony opening

- there are two zones where smoke dilution takes place: in the doorjet following the gaseous flow from the show-room to the walkway, and in the doorjet following the flow entering the atrium space from the same walkway

- atrium smoke vents do not operate very efficiently, since outside air flows through them into the atrium space. Consequently there is already a smoke layer of substantial size in the atrium space

- in spite of the fan-powered air supplies, there is no pressurization in the 4 th and 5 th-level walkways (observe the countercurrent flows through either balcony). 
(NB: Figure 4 arrows refer to various mass flow rates (flows through openings and entrained flows).

\section{PRESENTATION AND ANALYSIS OF STMULATION RESULTS}

Firstly, orders of magnitude of some significant variables are given:

- smoke penetration in atrium volume begins typically 1 minute after the fire outbreak. After a few minutes about half the atrium is smokelogged, as well as walkways adjacent to smoke-logged zones of the atrium volume

- the dilution rate of smoke flowing from the show-room to the upper layer of the walkway next to it is 2. From this walkway to the upper layer of the atrium it ranges from 10 to 50 (influence of plume heights on doorjet entrainment rates)

- mean temperature elevation in atrium volume ranges typically from a few degrees $C$ to ten times more.

Figures 5 and 6 refer to the same simulation as Figure 4, which illustrates the influence of initial ambient conditions presently summer conditions): here an additional smoke dilution process takes place, since outside air enters the top of atrium, until a moment of maximal smoke-logging (Figure 5), followed by an inversion of atrium/outside flows in natural ventilation (Figure 6).

Figures 7 to 9, displaying ambient conditions calculated $1000 \mathrm{~s}$ after a fire outbreak, refer to three simulations under winter conditions, with various choice of the level where fire breaks out: when this is the 1st level of the building, the atrium plume is very high, therefore the dilution rate of smoke is big and the atrium upper layer is thick and not very hot. Conversely, on Figure 9, with a thinner, hotter upper layer of smoke in atrium space, the 5 th level walkway remains protected from smoke penetration. In this case, it can be noticed that the process of smoke exhaust through upper vents of atrium is not really affected by the diminution of the upper layer thickness since higher temperatures counterbalance this diminution of thickness: smoke exhaust power thus remains sufficient.

Natural ventilation between the atrium volume and the outside plays an important role for smoke exhaust, and additional simulations (6) have shown that natural ventilation processes were generally not controlled essentially by temperature differences due to and maintained by existence of a big fire nearby, but were rather controlled by temperature differences due to initial conditions. This may lead however to troublesome problems under summex ambient conditions, for which firegenerated pressure fields must be influent enough in order to avoid complete smoke-logging of the building (that might occur in cas of small fire). Nevertheless, fan-powered processes of air supply to the atrium space have as well a non negligible influence on the evolution of initial conditions during the fire: they increase significantly the delays preceding the beginning of smoke-logging in "sensitive" spaces (atria and walkways) (6).

Existence of weaknesses in the computer model that was used for the simulations leads to the following remarks: 


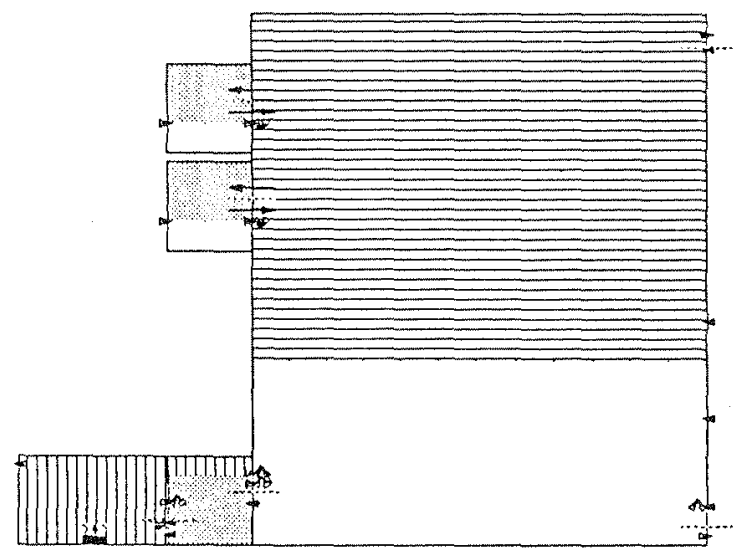

Figure 5

Same as figure 4, 330 sec after fire outbreak

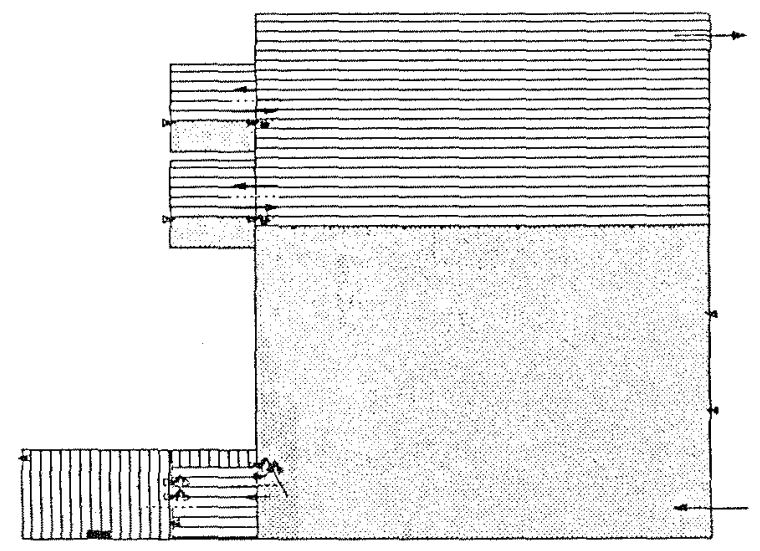

Figure 6

Same as Eigures 4 and 5 ,

half an hour after fire outbreak 


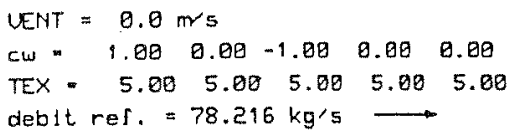

FIGURE 7

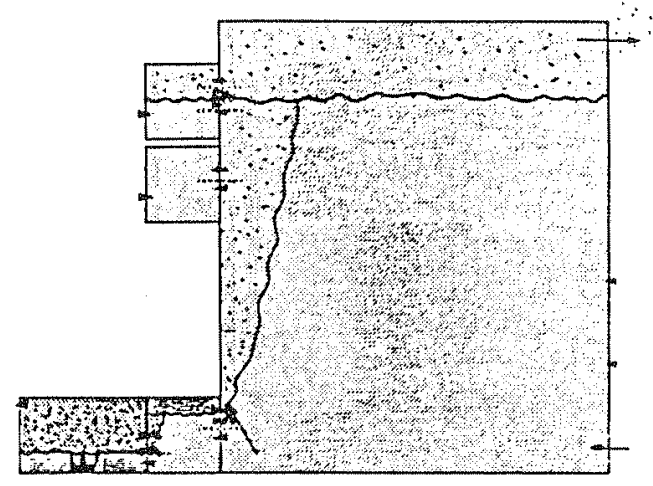

Cross-section of internal configuration calculated for winter conditions; examined $1000 \mathrm{~s}$ after fire outbreak

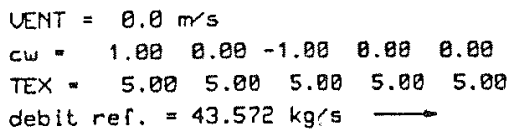

FIGURE 8

Calculated state for another simulation with winter conditions and another location of the fire room at $3 \mathrm{rd}$ level ( $1000 \mathrm{~s}$ after fire outbreak).

VENT $=0.0 \mathrm{~m} / \mathrm{s}$

$c_{W}=1.80 \quad 0.00-1.80 \quad 0.00 \quad 0.80$ TEX - $5.00 \quad 5.00 \quad 5.00 \quad 5.00 \quad 5.00$ debit ref. $=49.294 \mathrm{~kg} / \mathrm{s} \longrightarrow$

FIGURE 9

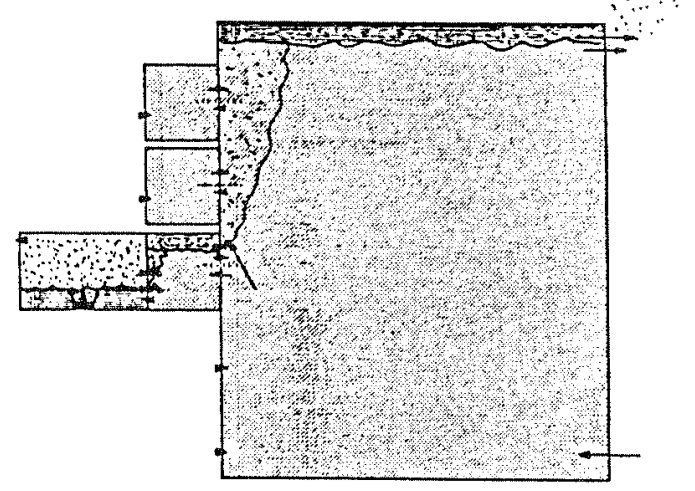


- when no smoke-logging is predicted, there might be local, diffuse smoke-logging processes (in the vicinity of smoke plumes developed in the atrium volume for instance)

- stratification of smoke under the atrium ceiling might not exist (e.g. when differences of temperatures are too small), therefore the atrium volume might be in some cases (e.g. small fire) fully logged with very diluted smokes

- initial stratification of ambient air, e.g. in the atrium volume, might complicate smoke-logging and smoke dilution processes.

\section{ADDITIONAL SIMULATIONS AND CONCLUSION}

In order to integrate the fact that the CNIT atrium building is fully sprinkled and that a fire brigade is located permanently near the building and could therefore begin to fight any fire after a few minutes, other simulations were performed, in which some description of fire source reduction versus time was incorporated (6). They showed that smoke-logging rates in the atrium space and in the walkways could never reach a critical level and that safe conditions were then likely to be maintained in the building. Consequently, it was eventually decided to leave balconies unglazed on all levels except on the 5 th level where glazing was supposed to be capable of preventing any difficulty in people egress processes in case of fire.

Although CSTB computer model CIFI is based on simple descriptions of physical phenomena, its capability of treating an environment involved in fire development and smoke propagation processes as a whole proved essential for the study of smoke movement and smoke control in the specific case of the CNIT atrium building.

As a matter of fact, the problem of ensuring safe environmental conditions in atrium buildings in case of fire cannot be satisfactorily solved but in a global manner, since atrium configurations always involve internal volumes widely open on each other. This certainly explains why above-mentioned capabilities of CIFI computer code could already contribute to a better understanding and assessment of processes of smoke movement in atrium buildings.

Nevertheless, because basic assumptions of zone models are very rough compared with real world configurations, any analysis of predicted results should always lead to cautious judgments, the only ones likely to contribute effectively to an improvement and even to an elaboration of a new intuition, thus enlarging the field of fire safety knowledge.

\section{ACKNOWIEDGEMENTS}

The present study was supported by SARI, which managed the recent CNIT redevelopment we are especially indebted to the Ministry of Research and to the "Direction de la securité Civile" of the French Ministry of the Interior for having facilitated the elaboration of the CIFI computer code, through the French research programme FIS (Eeu, Incendie, sécuritél. 
(1) "Réglement de sécurité contre l'Incendie relatif aux établissements recevant du public : dispositions génerales et commentaires officiels" du Ministère de l'Intérieur/Direction de la sécurité Civile, edited by "France sélection", $9-13$ rue du Département, 75925 PARIS CEDEX 19, Tél (33.1) 42081818

(2) Bodart, X.E., "Analyse de sous-procédures réglementaires, ...", 4 CSTB reports, July 1988

(3) DeCicco, P.R., "Life Safety Considerations in Atrium Buildings", SFPE Technology Report 82-3, Boston (Massachusetts), 1982

(4) Hansell, G.O., "Smoke Control in Atrium Buildings", colt International (UK) limited, 1987

(5) Note Technique $\mathrm{n}^{\circ} 263$, MI-DSC (Ministry of the Interior), 6 May 1987

(6) Bodart, X.E. and Curtat, M.R., "Risque d'enfumage de l'atxium du CNIT suite à un feu de boutique", CSTB Report, April 1989

(7) Curtat, M.R., "La modélisation physique des phénomènes de l'incendie : démarches actuelles, promesses et limites", Proceedings of the $5 \mathrm{th}$ Aquitanian Symposium of Hygiene and Safety, Bordeaux, October 1989

(8) Bodart, X.E., "La simulation numérique de l'enfumage de batiments", ibid

(9) Bodart, X.E. and Curtat, M.R., "Prediction of Entrained Mass flows through vertical openings in a Multiroom Fire Model", second international symposium of IAFSS, Tokyo, 1988

(10) Special issue of "Revue Générale de Thermique" (R. G. T.) about Erench research on fire safety, May 1988

(11) Zukoski and Kubota, "A Computer Model for fluid dynamic aspects of a transient fire", NBS report, June 1978, contract \#5-9004

(12) Thomas et al, "Investigation into the flow of hot gases in roof venting", Fire Research Technical Paper $n^{\circ} 7$, published by Hex Majesty's stationery office, UK, 1963 